\title{
An inventory of supershells in nearby galaxies: first results from THINGS
}

\author{
Ioannis Bagetakos ${ }^{1}$, Elias Brinks ${ }^{1}$, Fabian Walter ${ }^{2}$ and Erwin de Blok ${ }^{3}$ \\ ${ }^{1}$ Centre for Astrophysics Research, University of Hertfordshire, UK \\ ${ }^{2}$ Max-Planck-Institut für Astronomie, Heidelberg, Germany \\ ${ }^{3}$ Mount Stromlo Observatory, Australian National University, Canberra, Australia
}

\begin{abstract}
The HI Nearby Galaxy Survey (THINGS), is a 21-cm HI line survey of a sample of 34 nearby $(3-10 \mathrm{Mpc})$ galaxies (Walter et al. 2005). The observations were carried out with the VLA and have a velocity resolution of $5 \mathrm{~km} \mathrm{~s}^{-1}$ or better and an angular resolution of $7^{\prime \prime}$ which at this distance range corresponds to a linear resolution of 100-300 pc. One of the primary goals of THINGS is to look at the fine-scale structure of the Interstellar Medium (ISM) and examine how it varies as a function of Hubble type, star formation rate, galaxy mass, metallicity, etc. We present one of the first science results from this project, an inventory of HI shells in the galaxies NGC 628, NGC 3184, and NGC 6946.
\end{abstract}

Keywords. galaxies: ISM — ISM: structure - radio lines: ISM

\section{Results}

The morphology of the neutral ISM is greatly affected by massive stars through the combined effects of their stellar winds and, eventually, their demise as supernovae. Because massive stars tend to form in associations they will end their lives within the same, relatively short time span and within a small volume which leads to the formation of expanding bubbles of coronal gas in the ISM. These structures push the neutral gas outwards, compress it, and at least in some cases trigger secondary or induced star formation on the expanding rims. We have started the analysis of the structure of the ISM of all THINGS galaxies and some first results are given here. We find 19 well-defined HI shells in NGC 3184, 40 in NGC 6946 and 58 shells in NGC 628. The expansion velocities of these shells range from 5 to $20 \mathrm{~km} \mathrm{~s}^{-1}$ in all three galaxies. The distribution of the kinematical ages peaks at about the same value (40-60 Myr). The size distribution of the holes appears to be different in the three galaxies: NGC 3184 has the largest holes (mean $\sim 1140 \mathrm{pc}$ ) and NGC 6946 the smallest (mean $\sim 700 \mathrm{pc}$ ). All three galaxies are large, late-type (Sc or Scd) galaxies. The difference in the number and size of holes detected depends on many factors, such as the inclination of the galaxy, its proximity, its (past) star formation rate, etc. We expect that an analysis of all 34 galaxies in the THINGS sample will allow us to verify if there are indeed clear trends of the properties of the HI holes (size, expansion velocity, energy requirement and kinematical age) with those of the host galaxies (mass, metallicity, SFR, and Hubble type, among others).

\section{Reference}

Walter, F., Brinks, E., de Blok, W. J. G., Thornley, M. D., \& Kennicutt, R. C. 2005, First Results from THINGS: The HI Nearby Galaxy Survey (ASP-CS), 331, 269 